\title{
Keyboard Device
}

National Cancer Institute

\section{Source}

National Cancer Institute. Keyboard Device. NCI Thesaurus. Code C50018.

A device consisting of mechanical keys that are pressed to create input to a computer. 